
PRACTICE
CMAJ

\title{
Diffuse reticulate purpura in a boy with anorexia nervosa
}

\author{
Cristina Garrido Colmenero PhD, José Aneiros Fernández MD
}

\begin{abstract}
Competing interests: None declared.

This article has been peer reviewed.

The authors have obtained patient consent.

Affiliations: Department of Dermatology and Venereology (Garrido Colmenero); Department of Pathology (Aneiros Fernández), Virgen de Las Nieves Hospital, Granada, Spain
\end{abstract}

\section{Correspondence to:}

Cristina Garrido Colmenero, cristinagarrido86@gmail.com

CMAJ 2015. DOI:10.1503 /cmaj.141543

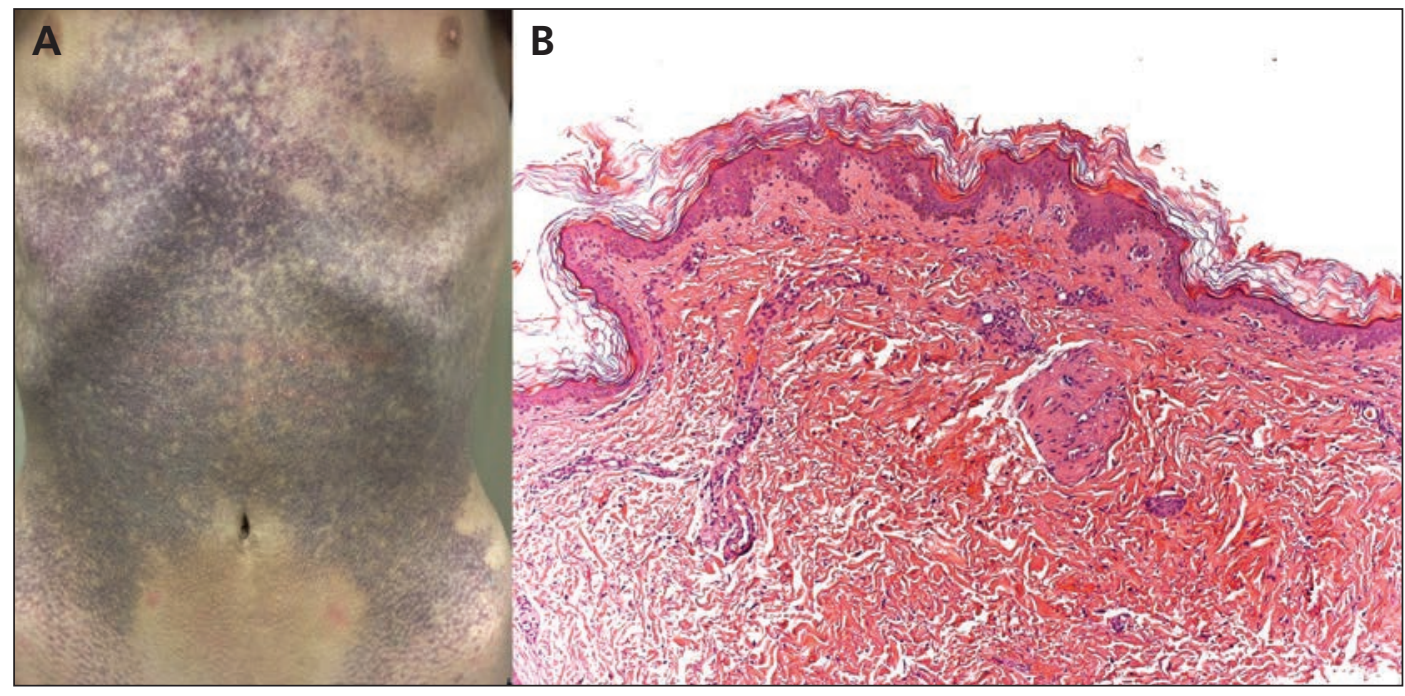

Figure 1: (A) Diffuse, reticulate, purpuric macules over the trunk of a 14-year-old boy with a 12-kg weight loss related to anorexia nervosa. (B) Histopathology of the reticulate purpura revealed dermal hemorrhage without vasculitis or vascular damage (hematoxylin-eosin, original magnification $\times 10$ ).

A 14-year-old boy presented to the emergency department with a one-week history of asymptomatic, diffuse, reticulate, purpuric macules over his trunk. The iliac crests were spared by the rash (Figure 1A). The patient's parents reported that he had lost $12 \mathrm{~kg}$ over the preceding two months from a baseline of about $50 \mathrm{~kg}$ (at a height of $160 \mathrm{~cm}$ ). He was bradycardic (49 beats/min) and hypotensive (96/47 $\mathrm{mm} \mathrm{Hg}$ ), with cold extremities and pitting edema of the lower legs. Our differential diagnosis included scurvy, vasculitis and coagulation abnormalities.

The patient's electrolyte levels, hemoglobin concentration, leukocyte count, albumin level, transaminase levels, coagulation profiles, serum vitamin $B_{12}$ level, folic acid level, ascorbic acid level and renal function were all within normal limits. Skin biopsy showed focal erythrocyte extravasation in the dermis without vasculitis or vascular damage (Figure 1B).

During the patient's subsequent hospital admission, we diagnosed anorexia nervosa in consultation with our psychiatric team. With adequate nutritional supply and energy intake, the patient gained weight, and after one month, the rash was gone. At a six-month follow-up, the rash had not returned, and the patient was still receiving psychiatric treatment

Severe and rapid weight loss is a critical problem in adolescence. In the context of severe malnutrition, the presence of a purpuric rash may be a warning of impending decompensation. ${ }^{1}$ It has been speculated that the rash is the result of bone marrow depression from starvation and subsequent thrombocytopenia. ${ }^{2}$ This explanation does not fit our patient's case, however, given his normal blood work. Weakening of capillary walls and dermal supporting structures related to malnutrition is a more plausible explanation for the findings in our patient. ${ }^{1,2}$ Although this striking rash is uncommon, medical practitioners may wish to keep it in mind as a potential manifestation of severe anorexia nervosa.

\section{References}

1. Pin-Chi C, Chih-Jung H, Hsien-Ching C. Diffuse reticulate purpura in anorexia nervosa. Dermatol Sinica 2003;21:68-74.

2. Strumia R. Eating disorders and the skin. Clin Dermatol 2013; 31:80-5. 